\section{Trends with benefits}

\section{By C. Simone Fishburn, Senior Editor}

The FDA set up its adverse event database decades ago to capture the side effects of marketed drugs, but a group from the Icahn School of Medicine at Mount Sinai has now exploited the millions of entries to extract a different type of information-beneficial drug combinations that statistically reduce a drug's risk of toxicity. The positive interactions could reveal nodes at which pathways intersect, providing new targets to help guide drug discovery.

"Our strategy was to ask what if combining drugs could have some good effects," team leader Ravi Iyengar told SciBX.

Using the FDA Adverse Event Reporting System (FAERS) database as its principal source, the group devised an approach to identify marketed drugs that produced fewer adverse events (AEs) when used in combination with other drugs than when used alone. ${ }^{1}$

Iyengar's goal was to find pairs of drugs with beneficial interactions, explore the networks associated with each drug's target and then find points of intersection that might separate therapeutic effects from AEs to help guide drug discovery.

Iyengar is a professor of pharmacology and systems therapeutics at Mount Sinai and director of Systems Biology Center New York.

\section{Better with Byetta}

The group focused its first search on Avandia rosiglitazone, a diabetes drug with a label that warns of an increased risk of myocardial ischemia and cardiovascular effects.

The team expected a high number of reported cases of Avandia AEs and hypothesized that the large numbers of diabetics taking multiple drugs would increase the odds of finding significant trends.

A scan of about 4 million entries in the FAERS database revealed about 63,064 reports of AEs among patients using Avandia. Of these, 20,005 were associated with myocardial infarction (MI), representing about $32 \%$ of the Avandia AEs.

The group then searched FAERS and identified 10 drugs that, when taken with Avandia, were associated with MI incidence substantially lower than $32 \%$.

Byetta exenatide emerged as the most effective drug in reducing the odds of Avandia-associated MI. The database contained 4,460 reports of AEs for patients taking both Byetta and Avandia, of which 95 were MIs. That $2 \%$ rate was comparable to the overall MI representation in FAERS.

Combinations of Avandia and Seroquel quetiapine or Diamicron gliclazide reduced the MI-associated reports to $7 \%$ and $5 \%$, although the sample size was small-each combination had less than 700 cases.

For another 7 drugs in combination with Avandia, the MI proportion ranged from $13 \%$ to $22 \%$ and the number of entries ranged from about 1,400 to 11,800 .

Avandia is marketed by GlaxoSmithKline plc. Diabetes drugs Byetta and Diamicron are sold by Bristol-Myers Squibb Co. and Servier, respectively. AstraZeneca plc markets Seroquel to treat schizophrenia and other neurological disorders.

\section{Networking}

Iyengar's group then focused on finding molecular mechanisms that might explain the link between Avandia drug combinations and reduced MI risk.

As a first step, the team used systems biology to create cell biological interaction networks centered around Avandia's target, peroxisome proliferation-activated receptor- $\gamma$ (PPARG; PPAR $\gamma$ ).

Next, the group focused on fluid retention and blood clotting as two plausible pathways by which Avandia might cause MI.

For each pathological process, the team searched for transcriptional relationships and protein-protein interactions connected with PPAR $\gamma$ and crafted a subnetwork that branched out from that target.

The team then looked for targets of the Avandia-interacting drugs within the two subnetworks and identified a node of convergence with Byetta in the clotting network at plasminogen activator inhibitor 1 (SERPINE1; PAI1).

Iyengar's group treated diabetic mice with Avandia and Byetta and found that the combination normalized Pail levels, and it decreased clotting dynamics compared with Avandia alone.

The researchers did not take the next step and use the PAI1 node to design new drugs, but they think the overall strategy can identify previously unknown pathway interactions that can be incorporated into drug discovery screens.

Data were reported in Science Translational Medicine.

\section{Strength in numbers}

Iyengar acknowledged the drawbacks of AE databases, as they rely on self-reporting and have no denominator such as the total number of prescriptions. As such, it is not possible to know how significant any interaction is within the total number of patients taking a particular combination of drugs.

Nevertheless, he told SciBX, the large size of FAERS compensates for the lack of a denominator, and the large numbers of cases fed into the statistics far exceed the numbers used in clinical trials.

In addition, according to Iyengar, the strength of the approach for identifying new targets or nodes of interaction is that the hypotheses derive from human clinical data rather than in vitro or cell-based experiments.

Thus, he thinks that the approach can yield information about drug combinations that could guide clinical practice. 


\section{ANALYSIS}

"The trick is to find the balance or mixture of drugs that will raise the therapeutic index," he told SciBX.

Whereas Byetta and Avandia are both used to treat diabetes, other drug combinations from diverse indications were also identified in the FAERS database search.

Indeed, Iyengar's group found more than 19,000 other combinations for which co-prescribing drugs could reduce the toxicity associated with one of them.

For example, a selective serotonin reuptake inhibitor (SSRI) and the histamine $\mathrm{H} 2$ receptor $(\mathrm{HRH} 2 ; \mathrm{H} 2 \mathrm{R}$ ) antagonist Zantac ranitidine significantly reduced the suicide rate from $3.1 \%$ for an SSRI alone to $0.6 \%$ for the combined drugs.

Iyengar said that pharmas could use the information from his team's screens to run prospective clinical trials to assess whether combinations might provide a significant therapeutic advantage over standard of care.
Mount Sinai has filed for patents covering data in the study, and the findings are available for licensing.

Fishburn, C.S. SciBX 6(44); doi:10.1038/scibx.2013.1245

Published online Nov. 14, 2013

\section{REFERENCES}

1. Zhao, S. et al. Sci. Transl. Med.; published online Oct. 9, 2013; doi:10.1126/scitransImed.3006548

Contact: Ravi lyengar, Icahn School of Medicine at Mount Sinai, New York, N.Y.

e-mail: ravi.iyengar@mssm.edu

\section{COMPANIES AND INSTITUTIONS MENTIONED}

AstraZeneca plc (LSE:AZN; NYSE:AZN), London, U.K. Bristol-Myers Squibb Co. (NYSE:BMY), New York, N.Y. Food and Drug Administration, Silver Spring, Md.

GlaxoSmithKline plc (LSE:GSK; NYSE:GSK), London, U.K. Icahn School of Medicine at Mount Sinai, New York, N.Y. Servier, Neuilly-sur-Seine, France

Systems Biology Center New York, New York, N.Y. 